\title{
The Economic Rationale for Integrated Tariffs in Local Public Transport
}

\author{
Carla Marchese* \\ Workshop Hermes "I servizi a rete tra monopolio e concorrenza: \\ trasporto pubblico ed energia", Moncalieri, November 26th, 2003
}

\begin{abstract}
In this paper it is shown that integrated tariffs can be used to extract the consumer's surplus when there are a lot of connections supplied, so that a law of large number applies in the estimation of the consumer's willingness to pay. The time validity limitations of tickets are explained by a nonlinear pricing approach. Links between optimal pricing in local public transport and network characteristics are highlighted.
\end{abstract}

\section{Introduction}

In many metropolitan areas public transport services adopt a multi-modal integrated fare system, with a flat fare structure. This paper examines the theoretical justifications for these "integrated tariffs". They are characterized by the following basic features:

- a fixed fee, that does not depend on the actual travel length;

- a given validity period of the travel document;

- the possibility of interchanging means of transport, e.g. tram, bus, underground train, etc.

A basic justification for integrated tariffs pertains to reducing the transaction costs involved in selling tickets, embarking passengers, providing price information, etc. These motivations have been pointed out in a stream of literature dealing with travelcards and the likes (see e.g. White [9], Carbajo[3], Gilbert and Jalilian [6]). If motivations based on transaction costs were the most relevant ones, however, the spreading of new technologies (e.g. contactless cards) should pave the way to the resort to prices more strictly tailored to

*Dept. of Public Policy and Public Choice Polis, Univ. of Eastern Piedmont, Via Cavour 84, 15100 Alessandria (Italy); Phone: +39-131-283718; fax: +39-131-283704; e-mail: carla.marchese@unipmn.it 
the travel length, the quality and comfort of transport, etc., while customer's payments should become more variable and more clearly linked to the specific characteristics of their consumption.

In this paper, however, we shall look for other reasons that may support integrated tariffs, by examining the applicability of modern price discrimination theory to the local collective transport fares. While models of two part tariffs and nonlinear pricing have been suggested with reference to travelcards (see e.g. Carbajo [3], Gilbert and Jalilian [5] and FitzRoy and Smith [4]), the focus was on providing explanations for quantity discounts given to users who make many trips, while no explanation was put forth for the resort to flat fares (e.g. hourly tickets) for small consumers.

The paper is organized as follows. In Section 2 a basic model of urban transport demand is presented, and integrated tariffs are motivated according to the large number approach suggested by Armstrong [1]. In Section 3 a nonlinear pricing approach is used to explain the resort to binding time constraints for consumers with low demand. In Section 4 the role of network and spatial characteristics in motivating the resort to integrated tariffs is considered. Some conclusions follow in Section 5.

\section{The basic model}

Let us assume that a monopolist renders available to the customers a list of journeys (each one linking an origin to a destination), which represent differentiated products. Differences arise on the basis of many possible factors, such as e.g. the relevance of the nodes connected by the trip, the length of the trip, the number of interchanges, etc.

Let us assume that each consumer aims at maximizing a quasi-linear utility function, under an income constraint:

$$
\begin{array}{rl}
\operatorname{Max}_{\mathbf{x}} & u(\boldsymbol{\alpha}, \mathbf{x})=u_{1}\left(\alpha_{1}, x_{1}\right)+u_{2}\left(\alpha_{2}, x_{2}\right), \ldots,+u_{n}\left(\alpha_{n}, x_{n}\right)+y \\
& \text { s.t. } \\
& y+P \leq M
\end{array}
$$

where $\boldsymbol{\alpha}$ is a vector of individual tastes, $\mathrm{x}$ a vectors of quantities ${ }^{1}$ of the $i=1 . . n$ transport products (i.e. journeys), $y$ is the agent's expenditure in goods other than transport, while $P$ is a flat fare for transport products. Functions $u_{i}$ are concave in $x_{i}$, while $u_{i}\left(\alpha_{i}, 0\right)=0$. Variables $\alpha_{i}$ could themselves be vectors. It is assumed that variables $\alpha_{i}$ are distributed according to independent distributions $F\left(\alpha_{1}\right), \ldots, F\left(\alpha_{n}\right)$. Moreover, the consumer does not bear any cost in terms of renunciation to alternative uses of time when travelling, nor has a binding time constraint: this assumption will be relaxed in Section 2.1. There are neither complementarity nor substitutability in demand. Income effects are

\footnotetext{
${ }^{1}$ While quantity (number of journeys of a given type) can vary only by discrete amounts, for the sake of simplicity it will be considered continuous.
} 
excluded by the quasi-linear specification of the utility function. The population size is normalized to 1 .

Let us assume that the fee $P$ does not encompasses the consumer's surplus that arises when (marginal) price applied to each transport product is zero. Thus the standard condition of equality of marginal benefits to marginal costs holds for each product, that is:

$$
u_{i}^{\prime}\left(\alpha_{i}, x_{i}\right)=0
$$

As (marginal) prices are zero for all the consumers, while demand does not depend on income, only tastes make a difference for the agent's choice. Thus the individual consumer's surplus $s_{i}\left(\alpha_{i}\right)$ for each product, partially or totally expropriated by the fee $P$, are distributed in the population according to the underlying distribution of $\alpha_{i}$.

Let us consider now the transport supply. For the sake of simplicity it is assumed that in the very short term only fixed costs are incurred in local transport production. This is a realistic assumption for a transport system that in the short run cannot adjust either capital or labor, and is not congested. While in this case efficiency might be reached by letting the service available for free, the need for financing the fixed cost can also be met efficiently by levying a fixed tax or fee on passengers (as long as no one drops out because of it).

Let us consider first of all the case in which the monopolist is perfectly informed about each consumer's tastes, so that she can resort to first degree price discrimination. For each service thus the discriminating monopolist collects full individual surpluses $s_{i}\left(\alpha_{i}\right)$ weighted by their probability of occurrence, and hence (as the population has been normalized to 1 ), she collects the average consumer's surplus of the population $\mu_{i}\left(\alpha_{i}\right)$. For the whole supply of products, the monopolist collects the total surplus $\mu=\mu_{1}+\mu_{2}, \ldots,+\mu_{n}$ enjoyed by consumers, summing over all the $n$ services provided as, given the independence assumption about the distribution of variables $\alpha_{i}$, mean total surplus is simply the sum of population mean surpluses for each service.

Armstrong [1] has noted that a monopolist who does not know the individual $\boldsymbol{\alpha}$ vectors, by introducing a fixed tariff, can simply aim at guessing the average consumer's surplus. While with reference to large numbers the error made in estimating the population mean total surplus is likely to be small, the main problem of this approach stays elsewhere. The larger the coefficient of variation of individual total surpluses, the more likely is that some consumers will find the fee too high, thus dropping out, while on the contrary some consumers will find it low and will be underexploited, thus driving the revenue downwards in comparison to the one collected by a perfectly discriminating monopolist. Armstrong [1], however, suggests that in some circumstances this difficulty can be overcome. Let us consider the worst version of the problem, that is a singleproduct (possibly fictitious) whose surplus distribution $F\left(s_{i}\left(\alpha_{i}^{*}\right)\right)$ has mean $\mu_{i}^{*}$ and variance $\sigma_{i}^{* 2}$, where $\mu_{i}^{*}$ is the smallest population mean surplus for a single product, while $\sigma_{i}^{* 2}$ is the largest variance. Let us consider that selling journey $i$ to an individual is like drawing one observation from the relevant distribution 
$F\left(s_{i}\left(\alpha_{i}\right)\right)$. To refer to the worst case in terms of profits, let us assume instead that the draw has been made from distribution $F\left(s_{i}\left(\alpha_{i}^{*}\right)\right)$. The larger the number of products supplied to the customer, the larger the number $n$ of draws from $F\left(s_{i}\left(\alpha_{i}^{*}\right)\right)$. Hence adding types of journeys supplied is like enlarging the sample. The coefficient of variation of total consumer's surplus, i.e. $\frac{\sqrt{n} \sigma_{i}^{*}}{n \mu_{i}^{*}}=\frac{\sigma_{i}^{*} / \sqrt{n}}{\mu_{i}^{*}}$, becomes smaller and smaller as the number $n$ of products supplied increases. This means that the possibility of exit of customers for overexploitation or the risk of underexploitation fades out ${ }^{2}$ when the list of products supplied becomes very long, as each consumer can arrange her own preferred mix of products, and the longer the list made available the more likely it is that everyone finds a suitable bundle for which she is willing to pay the fixed fee.

While a monopolist aims at fully expropriating the total surplus, a public firm or a regulator that sets fees in order to maximize consumer's welfare with the constraint of balancing the budget or of meeting a given revenue target will only look for a fee that raises the needed revenue. Larger errors in the evaluation of the surplus can thus be made without endangering the budget balance. Moreover, from an efficiency point of view, what matters is the possibility of a drop out of consumers because of overexploitation, while errors that imply an underexploitation of some consumers only imply distributive consequences. This means that from the efficiency point of view only a one tail test must be passed by the fixed tariff.

Within this approach the expiration term of the document validity, that in practice often characterizes integrated tariffs, has not the role of constraining consumption. It is only a device useful for measuring the consumer's surplus and periodically cash it.

\subsection{The opportunity cost of time}

A more realistic description of the consumer's problem must take into account that travelling implies an opportunity cost in terms of time. The consumer's problem thus becomes:

$$
\begin{aligned}
& \operatorname{Max}_{\mathbf{x}} u(\boldsymbol{\alpha}, \mathbf{x})=y+u_{1}\left(\alpha_{1}, x_{1}\right)+u_{2}\left(\alpha_{2}, x_{2}\right), \ldots,+u_{n}\left(\alpha_{n}, x_{n}\right) \\
& \text { s.t. } \\
& y+P+V \sum_{i=1}^{i=n} m_{i} x_{i} \leq M^{F}=M+V T
\end{aligned}
$$

where $M^{F}$ stands for the agent's full income, including the value of the time endowment $T$, evaluated at the opportunity ${ }^{3} \operatorname{cost} V$, and $m_{i}$ is time needed

\footnotetext{
${ }^{2}$ Armstrong [1], by assuming that the error made by the monopolist in estimating the mean total rent is itself an increasing function of the coefficient of variation, calculates a convergence of profits to the level corresponding to the first degree price discrimination at the rate of $\frac{1}{\sqrt[3]{n}}$.

Note also that $\frac{\sigma_{i}^{*} / \sqrt{n}}{n \mu_{i}^{*}}$ is, by construction, an overestimation of the true coefficient of variation of the total consumer's surplus.

${ }^{3} \mathrm{~V}$ can be interpreted as the agent's salary, while $T$ is a fixed amount of time to be used either for travelling or for working. For the sake of simplicity leisure is disregarded.
} 
to accomplish trip $i$, or from now on its time-price. With this formulation, the agent faces positive marginal prices (to be precise: opportunity costs) for travelling. She might even drop out because of a too high opportunity cost of time. For the agent who stays in, the following conditions must hold ${ }^{4}$ :

$$
\begin{aligned}
\frac{u_{i}^{\prime}\left(\alpha_{i}, x_{i}\right)}{u_{j}^{\prime}\left(\alpha_{j}, x_{j}\right)} & =\frac{m_{i}}{m_{j}} \\
y+P^{t}+V \sum_{i=1}^{i=n} m_{i} x_{i} & =M^{F}=M+V T
\end{aligned}
$$

In practice the opportunity cost of time $V$ seems likely to vary from one consumer to another. The wage rate is obviously a variable that positively affects it. Other variables too are likely to be relevant in the real world (e.g. age, profession, etc.). The higher the opportunity cost of time of a given consumer, the lower the demand for public transport. Also drop out must affect particularly those citizens who have a high opportunity cost of time.

In this new scenario the provision of a document whose price reflects the average consumer's surplus may involve costs in terms of drop-out of low demand consumers: thus the supplier faces a trade-off between the quest for a large patronage (which could be reached by a low fee) or a deeper exploitation of the large demand customers (which would involve the opposite choice).

\section{The pricing policy when agents are heteroge- neous}

To keep the pricing problem simple, let us assume that there are two agent's type, $r$ and $p$ or rich and poor ${ }^{5}$, so that in problem (5) one must consider either $V_{r}$ or $V_{p}$, according to the type, while $V_{r}>V_{p}$, that is rich agents have a high opportunity cost of time. This approach implies that the so called no-crossing of demands property holds, that is type $p$ consumer's surplus (net of time costs) is always larger than that of type $r$, for a given quantity of each product. It is assumed that the distribution of variable $V=V_{r}, V_{p}$, is independent on $\boldsymbol{\alpha}$. Population is normalized to 1 , while $\lambda$ is the $p$ share.

Let us consider the choices available to a monopolist who knows $\boldsymbol{\alpha}$ but cannot distinguish the rich from the poor.

Paralleling standard price discrimination theory, the monopolist might consider the policy that yields the largest profit among the following three, namely: a) resort to a service offer and a fixed fee targeted for the rich low demand consumers; b) sell only to (poor) large demand customers; c) sell to both, by offering two bundle/fee combinations aimed at sorting consumers according to

\footnotetext{
${ }^{4}$ It is still assumed in this section that the validity time of the travel document is not a binding constraint.

${ }^{5}$ In practice the two groups might differ in many dimensions (e.g. age, profession etc.). What matters here is that they differ in the opportunity cost of time.
} 
type. Let us assume, without loss of generality, as only the F.O.C.s will be discussed, that constant production costs are zero. Profits to be considered are thus:

$$
\begin{gathered}
\pi_{1}=(1-\lambda+\lambda n) P_{r}^{*}\left(\boldsymbol{\alpha}, \mathbf{x}_{L}^{*}\right) \\
\pi_{2}=\lambda P_{p}^{*}\left(\boldsymbol{\alpha}, \mathbf{x}_{H}^{*}\right) \\
\pi_{3}=(1-\lambda) P_{r}\left(\boldsymbol{\alpha}, \mathbf{x}_{L}\right)+\lambda P_{p}\left(\boldsymbol{\alpha}, \mathbf{x}_{H}\right)
\end{gathered}
$$

where $\mathbf{x}_{L}^{*}$ is the bundle specifically designed for the rich, $\mathbf{x}_{H}^{*}$ the bundle specifically designed for the poor, while $\mathbf{x}_{L}$ and $\mathbf{x}_{H}$ refer to bundles and fees so designed as to induce the self-selection of customers.

If the monopolist applies a fee equal to the low demand (rich) consumer's net surplus when the bundle is $\mathbf{x}_{L}^{*}$, that is the one the rich is willing to consume at zero marginal monetary price, all the customers buy the service and fully exploit it. Moreover, as the supplier is neither willing nor able to avoid multiple purchases by poor consumers, the latter could buy a multiple $n$ (where $n \geq 1$ is an integer number) of the small bundle designed for the rich (e.g. buy many tickets). This effect is likely to arise whenever the difference in the willingness to pay between the two groups is large. Profit is $\pi_{1}$.

If instead the monopolist chooses the bundle and the fee that fully expropriates large demand consumers at similar conditions the ensuing profit is $\pi_{2}$, where it is taken into account that only large demand consumers buy the service. Low demand consumers drop out because their net surplus is smaller than the requested fee. In the third case the monopolist resorts to nonlinear prices.

\subsection{Nonlinear pricing}

It is assumed in this Paragraph that the solution of a standard discrete multiproduct nonlinear pricing problem ${ }^{6}$ satisfies also the extra constraints that must be met when forms of arbitrage, through multiple purchases, can arise. Katz [7] shows that this case is possible even if not general.

In a standard non linear pricing problem, the low demand consumers are rationed, while only at the top there is no distortion in the quantity supplied. Instead, when repeated purchases can arise, distortions might be more widespread. To understand why, note that the monopolist can only exploit the difficulties that consumers find because of the discontinuities in the quantities they can purchase, as only integer multiples of the available packages are sold. The monopolist can thus extract rents through the supply of alternatives to repeated purchases that are more apt to precisely fulfill the consumer's needs. For example the monopolist could find it profitable, under given parameters' values, to supply quantities larger than the efficient one to the consumers less prone to buy the service. This choice might be justified if it helps in taxing the high demand consumers, who would risk overshooting when trying to reach the desired quantity through repeated purchases. By increasing the quantity supplied to

\footnotetext{
${ }^{6}$ For a more technical analysis see Mirman and Sibley [8] - who tackle a continuous problemand the literature quoted therein.
} 


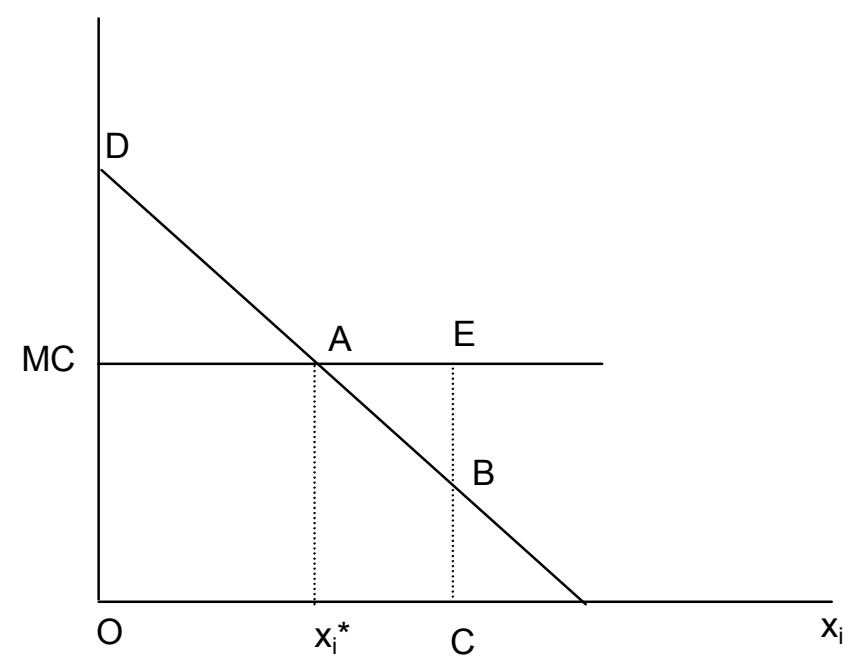

Figure 1: Oversupply

the low demand customer, the monopolist increases also the corresponding fee ${ }^{7}$, thus increasing the outlays of the consumers who make multiple purchases. For example, in Figure 1, the customer who buys quantity $C$ is ready in general to pay an amount equal to the area $O D B C$, which is larger than the amount paid when the efficient amount $x_{i}^{*}$ is supplied. The monopolist could be interested in supplying quantity $C$ in order to charge a quantity premium to a consumer demanding a larger quantity. In the transport model that we are considering, however, marginal cost (i.e. the opportunity cost of time) is borne personally by the consumer and not by the transport monopolist. Hence the monopolist, to encourage trips in excess of the efficient quantity from $x_{i}^{*}$ to $C$, should pay a subsidy $A E B$ to the customer, conditionally on the actual consumption of the extra quantity $x_{i}^{*} C$. This option seems both costly and far from real world experience.

Thus the relevant options in the model under consideration are either rationing the low demand consumers or supplying the efficient bundle to both types. The resort to forms of rationing for small demand consumers, and to quantity discounts for those who buy large quantities seems widespread in local

\footnotetext{
${ }^{7}$ For consumption above the efficient level, the (marginal) demand price is positive even if lower than the marginal cost.
} 
public transport. Hence, in the following, a typical case in which such a pattern arises is considered, that is the case in which the solution is the same as under the possibility of prohibiting multiple purchases.

The self selection constraints that must be met in this case are:

$$
\begin{aligned}
& s\left(\boldsymbol{\alpha}, \mathbf{x}_{H}\right)-P_{p}-V_{p} T_{H}>s\left(\boldsymbol{\alpha}, \mathbf{x}_{L}\right)-P_{r}-V_{p} T_{L} \\
& s\left(\boldsymbol{\alpha}, \mathbf{x}_{L}\right)-P_{r}-V_{r} T_{L}>s\left(\boldsymbol{\alpha}, \mathbf{x}_{H}\right)-P_{p}-V_{r} T_{H}
\end{aligned}
$$

where $s($.$) stands for the gross consumer's surplus, T_{H}=\sum_{i=1}^{i=n} m_{i} x_{i H}$ is total time spent for using the bundle $\mathbf{x}_{H}$ and $T_{L}$ is the corresponding term referred to bundle $\mathbf{x}_{L}$, while $m_{i}$ is time needed to accomplish trip $i$ which is available respectively in $x_{i H}$ or $x_{i L}$ quantities in each package. According to the standard procedure for these problems, only the downward constraint (6) with an equality sign will be taken into account, while the other one shall be checked ex-post. With reference to the participation constraint, satisfaction of the constraint for the low demand customer implies that the other one is automatically satisfied. The participation constraint, which must hold with equality as the monopolist benefits from expropriating the consumer's surplus (net of opportunity costs of time), is:

$$
P_{r}=s\left(\boldsymbol{\alpha}, \mathbf{x}_{L}\right)-V_{r} T_{L}
$$

By substituting (8) in (6), the downward self-selection constraint solves for:

$$
P_{p}=s\left(\boldsymbol{\alpha}, \mathbf{x}_{H}\right)-V_{p} T_{H}+T_{L}\left(V_{p}-V_{r}\right)
$$

Note that the fee $P_{p}$ to be applied to the large demand customer is lower than her net surplus, as the term in brackets is negative. The fee $P_{p}$ is lower the higher the opportunity cost of travelling for the low demand customers $V_{r}{ }^{8}$.On the other hand, $P_{p}>P_{r}$, due to the larger net surplus this type of agent enjoys when she consumes $\mathbf{x}_{H}^{*}$ instead of $\mathbf{x}_{L}^{*}$, as dictated by the self-selection constraint (6). The benefits due to the information rent for high demand customers refer only to the share of consumption corresponding to time $T_{L}$.

Let us now substitute prices into the monopolist objective function:

$$
\pi_{3}=(1-\lambda)\left[s\left(\boldsymbol{\alpha}, \mathbf{x}_{L}\right)-V_{r} T_{L}\right]+\lambda\left\{s\left(\boldsymbol{\alpha}, \mathbf{x}_{H}\right)-V_{p} T_{H}+T_{L}\left(V_{p}-V_{r}\right)\right\}
$$

By differentiating (10) with reference to the quantity of a single component $x_{i}$ of either bundle $\mathbf{x}_{L}$ or $\mathbf{x}_{H}$, we get:

$$
\begin{aligned}
\frac{\delta s\left(\boldsymbol{\alpha}, \mathbf{x}_{H}\right)}{\delta x_{i H}} & =V_{p} m_{i} \\
\frac{\delta s\left(\alpha, \mathbf{x}_{L}\right)}{\delta x_{i L}} & =V_{r} m_{i}+\frac{\lambda}{1-\lambda} m_{i}\left(V_{r}-V_{p}\right)=m_{i} \frac{1}{1-\lambda}\left(V_{r}-\lambda V_{p}\right)
\end{aligned}
$$

This is the standard result, that is for the large demand customer (i.e. the poor) the quantity supplied should be the efficient one, while for the low demand

\footnotetext{
${ }^{8}$ The explanation is that a high opportunity cost of time for the rich implies a low wilingness to pay on their part, which in its turn implies a stronger temptation of mimicking them for the poor.
} 
customer the quantity should be less than the efficient one. Note also that the difference between the prices for the two agent's types according to (11) does not depend on the tastes vector $\boldsymbol{\alpha}$, but only on the other parameters of the model. This property implies that a kind of constant mark-up must be applied to the time-price $m_{i}$ for the low demand customer. As, however, $m_{i}$ is not cashed by the firm (it is an opportunity cost borne by the traveller), collecting the mark-up in cash is likely to be problematic. Nevertheless, one can substitute the inflation, that must equally affect all time-prices, through a reduction of the total time that the agent can spend in travelling.

The quantity rationing to be applied to low demand consumers in order to realize the nonlinear pricing policy can thus also be implemented in time terms. That is, instead of explicitly defining the bundle of services made available, the monopolist offers a ticket with a validity time equal to the total time needed to consume bundle $\mathbf{x}_{L}$. The fee is set at a level such as to fully exploit the agent's net surplus when consumption is $\mathbf{x}_{L}$. Hence, according to this approach, the agent's problem includes also a binding validity time constraint. To induce the desired result, the monopolist must set the validity time in order to imply a shadow time-price that inflates $V_{r} m_{i}$ according to the r.h.s. of (11).

To check this approach, let us assume that the low demand consumer must satisfy the following further constraint, where $T_{v}$ stands for the validity time:

$$
\sum_{i=1}^{i=n} m_{i} x_{i L} \leq T_{v}
$$

let us solve for $y$ the income constraint in (5) to obtain:

$$
y=M+V_{r} T-P_{r}-V_{r} \sum_{i=1}^{i=n} m_{i} x_{i L}
$$

After substituting $y$ into the agent's utility function (3), the F.O.C. for the rich agent's utility maximization becomes:

$$
\frac{\delta u\left(\alpha, \mathbf{x}_{L}\right)}{\delta x_{i L}}=m_{i}\left(V_{r}+\mu_{r}\right)
$$

where $\mu_{r}$ is the Lagrange multiplier of the validity time constraint. When the rich consumer is in equilibrium at $\mathbf{x}=\mathbf{x}_{L}$, the r.h.s of (11) and that of (12) must be equal, in order to sustain the desired price discrimination and to maximize profits in (10).

With reference to poor consumers instead, as their choice need not be distorted, the offer may consist just in the possibility of consuming whichever amount the agent prefers, provided that she is ready to pay a fee equal to $P_{p}$ as defined in (9), without binding validity time limitations. A period of validity can all the same be established also for documents sold to poor customers, in order to ease the evaluation of the consumer's surplus and to periodically cash it. 
While the problem discussed refers to profit maximization, one could instead assume the point of view of a regulator and consider an objective function represented by:

$$
W=s_{r}+s_{p}+\beta \pi
$$

where $\beta<1$ is the weight assigned to profits. In this case it can be shown ${ }^{9}$ that bundle $\mathbf{x}_{L}$ depends also on $\beta$, which means that the validity time constraint $T_{v}$ shall be relaxed the more the lower the concern for profits.

The non-linear pricing approach provides a new possible explanation for the resort to (short and binding) validity periods for some travel documents, e.g. tickets: they provide a tool for implementing a price discrimination policy.

\subsection{Discussion}

Let us now drop the assumption of full information of the monopolist about $\boldsymbol{\alpha}$ and let us assume instead, as in Section 2, that the monopolist only knows the $\boldsymbol{\alpha}$ distribution. If the monopolist's best choice is that of targeting the supply to just one group of consumers (either the rich or the poor), the approach of fixing the fee with reference to the estimated average consumer's surplus, as suggested in Section 2, can be directly applied. The non-linear pricing approach instead implies specific further problems pertaining to the rationing to be applied to low demand customers and to the working of the self-selection when only an approximated version of the relevant prices is applied.

With reference to low-demand customers, if the coefficient of variation of their net rent when the short validity time document is available is large, some drop-out is likely to occur. No harm is caused instead by rich consumers who reach a surplus high enough to justify paying $P_{r}$ in a time shorter than $T_{L}$, as they simply do not fully exploit the validity time, but do not drop out.

It is also possible that some rich consumers, with over-the average willingness to pay, prefer the offer designed for the poor ones. But this is not a problem too, as the monopolist makes a larger profit when the agent pays the fee $P_{p}{ }^{10}$. The opposite case instead, that is poor customers with a surplus under the mean of their group, who prefer the offer designed for the rich, negatively affects profits.

However, a large number effect can be invoked also with reference to the non-linear pricing approach, as the time needed for poor customers to consume a suitable package and the willingness to pay within both groups will become closer and closer to their mean value in the population the longer the list of products supplied. That is, in this case too, the idea of guessing the true values is tenable as long as the supply of products is very wide: a more detailed explanation on this purpose is provided in the Appendix. Moreover, while in this paper it has been assumed that only fixed costs are borne to produce local public transport, the large-number non linear pricing approach, as shown by

\footnotetext{
${ }^{9}$ On this topic see Mirman and Sibley [8].

${ }^{10} \mathrm{It}$ is assumed that, nothwithstanding the reference to average data and the introduction of corrections aimed at underestimating the customer's willingness to pay, the ordering of the fees of the two groups (and of the profits made by the firm) is preserved, i.e. no pooling of the two groups arises.
} 
Armstrong [1], can be extended to cases of positive constant marginal costs for the supplier.

In a more realistic scenario, the customers of public transport can be classified in more than two types. While transport companies are likely to find it profitable to leave out some very low demand consumers, the travel documents provide many combinations of validity time and fee, with validity constraints which become less and less binding as the fee increases.

\section{The spatial determinants of demand}

The rationale suggested in this paper for integrated tariffs is based on the supply of equal opportunities to the customers. While this equality hinges to some extent upon subjective evaluations (the taste vector) it is also likely to be fostered by objective characteristics of available trips, that must be enough varied in order to meet differences in tastes. To clarify this point let us consider Figure 2 part a), where a nine nodes fully interconnected network is depicted, and assume that there are nine consumers, located (either because they leave there or arrive there from outside) at each node. Everyone can leave from her location and reach another node, either directly or passing through other nodes. This network type is also likely to be associated to a spatial organization whose nodes are equivalent in terms of economic functions, so that there is no specific need that pushes consumers located at a specific node to demand longer trips than other ones. Similar opportunities are thus offered on an objective basis. While actually chosen consumption bundles are likely to be different (one could expect for example that everyone demands preferentially the connections starting from her location), it seems reasonable to expect that similar consumer's surpluses arise. Differences in consumer's surplus will depend only on income or social characteristics, as assumed in Section 3.1. Hence there are favorable conditions for resorting to pricing policies that assign a relevant role to integrated tariffs.

If instead the network is more of the hub-and spoke type, as in Figure 2 part $b$, opportunities offered in the periphery will be less in terms of accessibility, where the three central nodes enjoy more links starting there. On the other hand, one may expect that the utility enjoyed per trip is higher ceteris paribus for periphery residents, as in such a network the availability of other non-transport services is likely to be lower in the periphery than in the hub, thus busting the willingness to pay for transport that links the periphery to the centre. In this case it seems advisable for the supplier to resort to an approach based on zoning (in the example centre and periphery), with heavier fees for passengers who travel in more than one zone, that almost invariably shall be the periphery residents or those whose traffic originates in the periphery.

These examples suggest that we should observe fully integrated tariffs based only on validity time and not on trip length in urban areas very homogenous in terms of economic relevance, and with fully connected transport networks. Integration among transport modes obviously contributes to characterize such a case. 


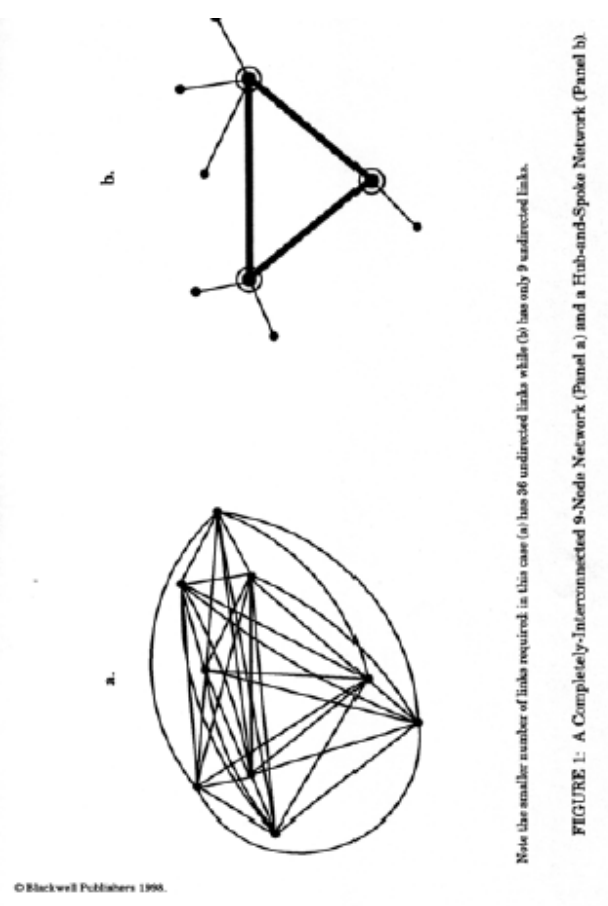

Figure 2: source: Bryan and O'Kelly [2]

On the other hand the more hierarchical is the spatial organization, the more unequal are the opportunities made available, the larger are the differences in the willingness to pay according to traffic origin and the lower the appeal of integrated tariffs. Tariff integration can be pursued with reference to specific segments of users (e.g. the travellers in the central city). The extensions of the approach to other areas implies on the one hand a suitably designed fee system that takes into account the aforementioned differences in the willingness to pay, and on the other hand a large enough transport supply (in terms of variety of available relevant trips) for all the segments of users involved, in order to justify the large number approach to pricing suggested above.

\section{Conclusions}

Integrated tariffs combine a large consumption flexibility for the consumer, who can choose among many products at zero marginal monetary price, with the possibility of exploitation of the consumer's willingness to pay for the supplier. Profits in the limit are not far from those of a fully discriminating monopolist. 
The restricted validity time that characterizes the travel documents with integrated tariffs contributes to measuring the consumer's surplus; moreover, in specific cases, it can provide the basis for sustaining a nonlinear pricing system.

Integrated tariffs, however, are more easily supported by homogeneous, highly integrated and fully connected transport networks, while they may involve negative effects in terms of passengers drop out or of willingness to pay underexploitation if the network does not posses the aforementioned characteristics.

The evolution of the technology of fees' collection is likely to provide the suppliers in the near future with a lot of information about user's characteristics, and to sharply reduce transaction costs. As, however, the patronage of each service varies over time, while also individual behavior changes in response to many stimuli, it seems unlikely that the personalization of fees (in order to exactly capture, in full or partially, each consumer's surplus) can be pushed too far. Data made available by the new collection systems can instead be used to better characterize consumer's types and to give a more robust basis to integrated tariffs, when the conditions of profitability discussed above are verified. Moreover, the reaction of consumers to a complicated price system can be negative, as it implies high costs of information and planning on their part, only marginally reduced by new collection systems. Hence the future of integrated tariffs seems still promising.

\section{Appendix}

Write:

$$
\begin{aligned}
\theta_{L} & =\mathbf{E}\left[T_{L}(\boldsymbol{\alpha})\right] ; \quad \mu_{L}=\mathbf{E}\left[s_{L}(\boldsymbol{\alpha})\right] ; \\
\theta_{H} & =\mathbf{E}\left[T_{H}(\boldsymbol{\alpha})\right] ; \quad \mu_{H}=\mathbf{E}\left[s_{H}(\boldsymbol{\alpha})\right] \\
\sigma_{L}^{2} & =\operatorname{Var}\left[s_{L}(\boldsymbol{\alpha})\right] ; \sigma_{H}^{2}=\operatorname{Var}\left[s_{H}(\boldsymbol{\alpha})\right]
\end{aligned}
$$

where $\theta_{L}$ is the expected value of $T_{L}, \mu_{L}$ is the expected value of the consumer's rent when $\theta_{L}$ is the validity time, $\theta_{H}$ is the expected travel time when the opportunity cost of time is $V_{p}$ and no validity time constraint is binding, $\mu_{H}$ is the corresponding expected rent.

The fees applied when the monopolist is not informed about the agent's $\boldsymbol{\alpha}$ are:

$$
\begin{gathered}
A_{L}=(1-\epsilon) \mu_{L}-(1+\epsilon) V_{r} \theta_{L} \\
A_{H}=(1-\epsilon) \mu_{H}-(1+\epsilon) V_{p} \theta_{H}+\theta_{L}\left[(1-\epsilon) V_{p}-(1+\epsilon) V_{r}\right]
\end{gathered}
$$

Let us call $S$ the set of consumers characterized by:

$$
\boldsymbol{\alpha} \mid(1-\epsilon) \mu_{L} \leq s_{L}(\boldsymbol{\alpha}) \leq(1+\epsilon) \mu_{L} \text { and }(1-\epsilon) \mu_{R} \leq s_{R}(\boldsymbol{\alpha}) \leq(1+\epsilon) \mu_{R}
$$

where $s_{R}(\boldsymbol{\alpha})=s_{H}(\boldsymbol{\alpha})-s_{L}(\boldsymbol{\alpha})$ while $\mu_{R}=\mu_{H}-\mu_{L}$ is the expected value of $s_{R}(\boldsymbol{\alpha})$. The probability of not satisfying each of the conditions provided in (13) 
is respectively:

$$
\begin{aligned}
& \operatorname{prob}\left\{\left|s_{L}(\boldsymbol{\alpha})-\mu_{L}\right| \geq \epsilon \mu_{L}\right\} \leq \frac{\sigma_{L}^{2}}{\epsilon^{2} \mu_{L}^{2}} \\
& \operatorname{prob}\left\{\left|s_{R}(\boldsymbol{\alpha})-\mu_{R}\right| \geq \epsilon \mu_{R}\right\} \leq \frac{\sigma_{R}^{2}}{\epsilon^{2} \mu_{R}^{2}}
\end{aligned}
$$

Note also that (14) plus (15) imply that:

$$
\operatorname{prob}\left\{\left|s_{H}(\boldsymbol{\alpha})-\mu_{H}\right| \geq \epsilon \mu_{H}\right\} \leq \frac{\sigma_{H}^{2}}{\epsilon^{2} \mu_{H}^{2}}
$$

Let us first consider the poor, large demand customers. The participation constraint is automatically satisfied whenever they belong to the set $S$. The downward self-selection constraint becomes:

$$
S_{H}(\boldsymbol{\alpha})-A_{H}-V_{p} T_{H}(\boldsymbol{\alpha}) \geq S_{L}(\boldsymbol{\alpha})-A_{L}-V_{p} \theta_{L}
$$

where, as already mentioned, it is taken into account that in this model agents (for each given $\boldsymbol{\alpha}$ ) do not differ in preferences (and hence the poor agent has the same rent as the rich one when they choose the small package), but only in opportunity cost of time. By substituting the fees into (17), one gets:

$$
S_{R}(\boldsymbol{\alpha})-(1-\epsilon) \mu_{R}+(1+\epsilon) V_{p} \theta_{H}-V_{p} T_{H}(\boldsymbol{\alpha})+\epsilon V_{p} \theta_{L} \geq 0
$$

While for agents belonging to the set $S$ it follows that $S_{R}(\boldsymbol{\alpha})-(1-\epsilon) \mu_{R} \geq 0$, to ensure that the whole condition (18) is satisfied one must exclude also cases in which $(1+\epsilon) V_{p} \theta_{H}<V_{p} T_{H}(\boldsymbol{\alpha})$. Violations of the latter requirement occur with probability:

$$
\operatorname{prob}\left\{\left|T_{H}(\boldsymbol{\alpha})-\theta_{H}\right| \geq \epsilon \mu_{H}\right\} \leq \frac{\sigma_{\theta}^{2}}{\epsilon^{2} \mu_{\theta}^{2}}
$$

where the absolute value of the difference is considered in order to exclude also cases of underexploitation of the consumer's rent due to an overestimation of the time spent in travelling.

Hence the expected profit from high demand consumers is as follows:

$\lambda\left[1-\frac{1}{\epsilon^{2}}\left(\frac{\sigma_{L}^{2}}{\mu_{L}^{2}}+\frac{\sigma_{R}^{2}}{\mu_{R}^{2}}+\frac{\sigma_{\theta}^{2}}{\mu_{\theta}^{2}}\right)\right]\left[(1-\epsilon) \mu_{H}-(1+\epsilon) V_{p} \theta_{H}+\theta_{L}\left((1-\epsilon) V_{p}-(1+\epsilon) V_{r}\right)\right]$

to be compared with a benchmark represented by:

$$
\lambda\left\{\mu_{H}-V_{p} \theta_{H}+\theta_{L}\left(V_{p}-V_{r}\right)\right\}
$$

When the list of products becomes long, the coefficients of variation that appears in (20) shrink ${ }^{11}$, thus reducing the gap with respect to (21).

\footnotetext{
${ }^{11}$ Substituted by an estimate of their value based upon the worst possible case referred to a single product, as suggested in the text.
} 
With reference to the rich customers, if they choose the poor's fee the firm's profit increases. Hence, keeping into account the participation constrain, a lower bound for the expected profit from the rich consumers is:

$$
(1-\lambda)\left(1-\frac{\sigma_{L}^{2}}{\epsilon^{2} \mu_{L}^{2}}\right)\left[(1-\epsilon) \mu_{L}-(1+\epsilon) V_{r} \theta_{L}\right]
$$

to be compared with a benchmark expected profit given by:

$$
(1-\lambda)\left(\mu_{L}-V_{r} \theta_{L}\right)
$$

Here again an argument based upon the large number approach can be applied to predict a shrinking of the difference between (22) and (23).

\section{References}

[1] Armstrong, M., 1999, Price Discrimination by a Many-Product Firm, Review of Economic Studies 66, 151-68.

[2] Bryan, D. and O'Kelly, M., 1999, Hub-and-spoke Networks in Air Transportation: an Analytical Review, Journal of Regional Science 39, 275-95.

[3] Carbajo, J.,1988, The Economics of Travel Passes, Journal of Transport Economics and Policy 22, 153-73.

[4] FitzRoy, F. and Smith I., 1999, Season Tickets and the Demand for Pubic Transport, Kyklos 52, 219-38.

[5] Gilbert, C.L. and Jalilian H., 1991, What Is a Farecard Worth?, Applied Economics 23, 1053-58.

[6] Gilbert, C.L. and Jalilian H., 1991, The Demand for Travel and for Travelcards on London Regional Transport, Journal of Transport Economics and Policy 25, 3-29.

[7] Katz, M. L., 1984, Nonuniform Pricing with Unobservable Numbers of Purchases, Review of Economic Studies 51, 461-70.

[8] Mirman, L. J. and Sibley, D., 1980, Optimal Nonlinear Prices for Multiproduct Monopolies, Bell Journal of Economics, 11, 659-70.

[9] Whilte, P.R., 1981,"Travelcard" Tickets in Urban Public Transport, Journal of Transport Economics and Policy 15, 17-34. 